Проблеми математичного моделювання

та теорії диференціальних рівнянь

УДК 517.977.56

\title{
ВАРІАЦІЙНІ РОЗВ'ЯЗКИ ЗАДАЧІ ОПТИМАЛЬНОГО КЕРУВАННЯ 3 НЕОБМЕЖЕНИМИ КОЕФІЩЕНТАМИ
}

\author{
${ }^{*}$ C. О. Горбонос, ${ }^{*} *$ П. I. Когут
}

* Дніпропетровсъкий начіоналъний університет, кафедра диференчіалъних рівнянь, вул. Казакова, 18/14, ДНУ, 49010, Дніпропетровсък, E-таil: gorbonos.so@gmail.com

** Дніпропетровський національний університет, кафедра диференціальних рівнянь, вул. Казакова, 18/14, ДНУ, 49010, Дніпропетровськ, E-mail: p.kogut@i.uа

Досліджено задачу оптимального керування параболічною системою з необмеженими коефіцієнтами. Введено поняття варіаційного розв'язку поставленої задачі керування та встановлено умови його існування.

Ключові слова: задача оптимізації, слабкий розв'язок, кососиметричні матриці, варіаційний розв'язок.

\section{1. Вступ}

У статті досліджується задача оптимального керування параболічною системою з необмеженими коефіцієнтами. Особливість даного класу задач полягає в тому, що матриця потоку є кососиметричною, а її елементи належать простору $L^{2}(\Omega)$. Показано, що відповідна початково-крайова задача $\in$ некоректною. Залучаючи $L^{\infty}$-апроксимацію матриці потоку та метод Гальоркіна, отримали умови, за яких оптимальні розв'язки вихідної задачі оптимального керування можна наблизити розв'язками відповідних апроксимаційних задач.

\section{2. Попередні результати та позначення}

У даному розділі наведемо основні позначення, поняття та факти з функціонального аналізу, необхідні для подальшого розгляду поставленої задачі.

Нехай $\Omega$ - відкрита обмежена з ліпшицевою межею підмножина простору $\mathbb{R}^{N}$, де $N \geq 2$. Нехай межа $\Omega$ складається із двох частин, які не перетинаються, тобто $\partial \Omega=\Gamma_{1} \cup \Gamma_{2}$. Множини $\Gamma_{1}$ і $\Gamma_{2} \in(N-1)$-вимірними. Надалі через $|E|$ будемо позначати міру Лебега $\mathcal{L}^{N}(E)$ будь-якої вимірної множини $E \subset \Omega . \chi_{E}-$ характеристична функція множини $E \subset \Omega$, тобто $\chi_{E}(x)=1$, якщо $x \in E$ і $\chi_{E}(x)=0$, якщо $x \notin E$.

Через $\mathbb{S}^{N}$ будемо позначати множину всіх кососиметричних матриць $C=$ $\left[c_{i j}\right]_{i, j=1}^{N}$, тобто $C$ - це квадратна матриця така, що $c_{i j}=-c_{j i}$, а отже $c_{i i}=0$.

(C) С. О. Горбонос, П. I. Когут, 2013 
Слід зауважити, що множина $\mathbb{S}^{N}$ може бути утотожнена з евклідовим простором $\mathbb{R}^{\frac{N(N-1)}{2}}$. Нехай $L^{2}(\Omega)^{\frac{N(N-1)}{2}}=L^{2}\left(\Omega ; \mathbb{S}^{N}\right)$ - простір вимірних інтегровних в квадраті функцій, значеннями яких $€$ кососиметричні матриці. Цей простір будемо наділяти нормою

$$
\|A\|_{L^{2}\left(\Omega ; \mathbb{S}^{N}\right)}=\left(\int_{\Omega}\left(\max _{\substack{i, j=1, \ldots, N \\ j>i}}\left|a_{i j}(x)\right|\right)^{2} d x\right)^{1 / 2} .
$$

Уведемо поняття варіаційної збіжності задач оптимального керування. Нехай

$$
\min \left\{I_{\varepsilon}(u, y):(u, y) \in \Xi_{\varepsilon}\right\}
$$

є параметризованою задачею оптимального керування, де $\varepsilon-$ малий параметр, $I_{\varepsilon}: \mathbb{U}_{\varepsilon} \times \mathbb{Y}_{\varepsilon} \rightarrow \overline{\mathbb{R}}-$ функціонал вартості, $\mathbb{Y}_{\varepsilon}-$ простір станів, $\mathbb{U}_{\varepsilon}-$ простір керувань, а $\Xi_{\varepsilon}-$ множина всіх допустимих пар. Далі кожну задачу оптимального керування (2.1) пов'яжемо з відповідною задачею умовної оптимізації:

$$
\left\langle\inf _{(u, y) \in \Xi_{\varepsilon}} I_{\varepsilon}(u, y)\right\rangle \text {. }
$$

Припустимо, що існує банахів простір $\mathbb{U} \times \mathbb{Y}$, відносно якого $\in$ означеною $\tau$-збіжність у шкалі просторів $\left\{\mathbb{U}_{\varepsilon} \times \mathbb{Y}_{\varepsilon}\right\}_{\varepsilon>0}$.

Означення 2.1. Задача $\left\langle\inf _{(u, y) \in \Xi} I(u, y)\right\rangle$ називається варіаційною границею послідовності (2.2) при $\varepsilon \rightarrow 0$

$$
\left\langle\inf _{(u, y) \in \Xi_{\varepsilon}} I_{\varepsilon}(u, y)\right\rangle \underset{\varepsilon \rightarrow 0}{\stackrel{\operatorname{Var}}{\longrightarrow}}\left\langle\inf _{(u, y) \in \Xi} I(u, y)\right\rangle
$$

тоді і тільки тоді, коли виконуються умови:

(а) Відносно шкали просторів $\left\{\mathbb{U}_{\varepsilon} \times \mathbb{Y}_{\varepsilon}\right\}_{\varepsilon>0}$ простір $\mathbb{U} \times \mathbb{Y}$ задовольняє властивість: для будь-якого $\delta \geq 0$ і для кожної пари $(u, y) \in \Xi$ існує пара $\left(u^{*}, y^{*}\right) \in \Xi$ і послідовність $\left\{\left(u_{\varepsilon}, y_{\varepsilon}\right) \in \mathbb{U}_{\varepsilon} \times \mathbb{Y}_{\varepsilon}\right\}_{\varepsilon>0}$ такі, що

$$
\left\|u-u^{*}\right\|_{\mathbb{U}}+\left\|y-y^{*}\right\|_{\mathbb{Y}} \leq \delta \quad \text { і } \quad\left(u_{\varepsilon}, y_{\varepsilon}\right) \stackrel{\tau}{\longrightarrow}(u, y) \quad \text { в } \quad \mathbb{U}_{\varepsilon} \times \mathbb{Y}_{\varepsilon} .
$$

(аа) Якщо послідовності $\left\{\varepsilon_{k}\right\}_{k \in \mathbb{N}}$ i $\left\{\left(u_{k}, y_{k}\right)\right\}_{k \in \mathbb{N}}$ такі, що $\varepsilon_{k} \rightarrow 0$ при $k \rightarrow \infty$, $\left(u_{k}, y_{k}\right) \in \mathbb{U}_{\varepsilon_{k}} \times \mathbb{Y}_{\varepsilon_{k}}$ для будь-якого $k \in \mathbb{N} \mathrm{i}\left(u_{k}, y_{k}\right) \stackrel{\tau}{\longrightarrow}(u, y)$ в $\mathbb{U}_{\varepsilon_{k}} \times \mathbb{Y}_{\varepsilon_{k}}$, тоді

$$
(u, y) \in \Xi ; \quad I(u, y) \leq \liminf _{k \rightarrow \infty} I_{\varepsilon_{k}}\left(u_{k}, y_{k}\right)
$$

(ааа) Для кожної пари $(u, y \in \Xi) \subset \mathbb{U} \times \mathbb{Y}$ і будь-якого $\delta>0$ існує стала $\varepsilon^{0}>0$ і послідовність $\left\{\left(u_{\varepsilon}, y_{\varepsilon}\right)\right\}_{\varepsilon>0}$ такі, що

$$
\left(u_{\varepsilon}, y_{\varepsilon}\right) \in \Xi_{\varepsilon}, \quad \forall \varepsilon \leq \varepsilon^{0}, \quad\left(u_{\varepsilon}, y_{\varepsilon}\right) \stackrel{\tau}{\longrightarrow}(\widehat{u}, \widehat{y}) \text { в } \Xi_{\varepsilon},
$$




$$
\begin{gathered}
\|u-\widehat{u}\|_{\mathbb{U}}+\|y-\widehat{y}\|_{\mathbb{Y}} \leq \delta, \\
I(u, y) \geq \limsup _{\varepsilon \rightarrow 0} I_{\varepsilon}\left(u_{\varepsilon}, y_{\varepsilon}\right)-\widehat{C} \delta,
\end{gathered}
$$

де стала $\widehat{C}>0$ не залежить від $\delta$.

Тоді має місце такий результат.

Теорема 2.1. Нехай задача умовної оптимізаціӥ

$$
\left\langle\inf _{(u, y) \in \Xi_{0}} I_{0}(u, y)\right\rangle
$$

е варіачійною граничею послідовності (2.2) в сенсі означення 2.1. Нехай ияя задача мае единий розв'язок $\left(u_{0}, y_{0}\right) \in \Xi_{0} i$ нехай для кожного $\varepsilon>0$ пара $\left(u_{\varepsilon}^{0}, y_{\varepsilon}^{0}\right) \in \Xi_{\varepsilon}$ є мінімізантом функціонала $I_{\varepsilon}$ на відповідній множині $\Xi_{\varepsilon}$. Якщо послідовність $\left\{\left(u_{\varepsilon}^{0}, y_{\varepsilon}^{0}\right)\right\}_{\varepsilon>0}$ компактна відносно $\tau$-збіжності в $\left\{\mathbb{U}_{\varepsilon} \times\right.$ $\left.\mathbb{Y}_{\varepsilon}\right\}_{\varepsilon>0}, m o$

$$
\begin{gathered}
\left(u_{\varepsilon}^{0}, y_{\varepsilon}^{0}\right) \stackrel{\tau}{\longrightarrow}\left(u^{0}, y^{0}\right) \quad \text { в }\left\{\mathbb{U}_{\varepsilon} \times \mathbb{Y}_{\varepsilon}\right\}_{\varepsilon>0}, \\
\inf _{(u, y) \in \Xi_{0}} I_{0}(u, y)=I_{0}\left(u^{0}, y^{0}\right)=\lim _{\varepsilon \rightarrow 0} I_{\varepsilon}\left(u_{\varepsilon}^{0}, y_{\varepsilon}^{0}\right)=\lim _{\varepsilon \rightarrow 0} \inf _{\left(u_{\varepsilon}, y_{\varepsilon}\right) \in \Xi_{\varepsilon}} I_{\varepsilon}\left(u_{\varepsilon}, y_{\varepsilon}\right) .
\end{gathered}
$$

Нехай $V$ - простір Соболєва. Позначимо через $C([0, T], V)-$ банахів простір, який складається з усіх неперервних функцій $u:[0, T] \rightarrow V$ та який наділено нормою

$$
\|u\|_{C([0, T], V)}=\max _{0 \leq t \leq T}\|u(t)\|_{V} .
$$

Для $1 \leq p<\infty$ через $L^{p}([0, T], V)$ будемо позначати множину всіх вимірних функцій $u:[0, T] \rightarrow V$ таких, що

$$
\|u\|_{L^{p}([0, T], V)}=\left(\int_{0}^{T}\|u(t)\|_{V}^{p} d t\right)^{1 / p}<\infty .
$$

Зауважимо, що простір $L^{2}([0, T], V)$ є гільбертовим відносно скалярного добутку

$$
(u, v)_{L^{2}([0, T], V)}=\int_{0}^{T}(u(t), v(t))_{V} d t .
$$

\section{3. Постановка задачі оптимального керування}

Нехай об'єктом керування виступає така початково-крайова задача:

$$
\begin{array}{ccc}
y_{t}-\operatorname{div}(\nabla y+A(x) \nabla y)=f \quad \text { на } \quad \Omega \times[0, T] \\
y=0 \quad \text { на } \quad \Gamma_{1} \times[0, T], \quad \partial y / \partial \nu_{A}=u \quad \text { на } \quad \Gamma_{2} \times[0, T], \\
y\left(t_{0}, x\right)=y_{0} \in L^{2}(\Omega) \quad \text { на } \Omega,
\end{array}
$$


де $f \in L^{2}\left(0, T ; H^{-1}(\Omega)\right), u \in L^{2}\left(0, T ; L^{2}\left(\Gamma_{2}\right)\right)$ і $A(x) \in L^{2}\left(\Omega ; \mathbb{S}^{N}\right)$. Тоді задача оптимального керування полягає в тому, щоб знайти таку допустиму пару $(u, y)$, на якій функціонал вартості

$$
I(u, y)=\left\|y-y_{d}\right\|_{L^{2}\left(0, T ; H_{0}^{1}(\Omega)\right)}+\|u\|_{L^{2}\left(0, T ; L^{2}\left(\Gamma_{2}\right)\right)} \rightarrow \inf
$$

досягав би свого найменшого можливого значення при обмеженнях (3.1)(3.3).

Уведемо поняття слабкого розв'язку для задачі (3.1)-(3.3).

Означення 3.1. Слабким розв'язком задачі (3.1)-(3.3) для фіксованого керування $u \in L^{2}\left(0, T ; L^{2}\left(\Gamma_{2}\right)\right)$ і заданих $f \in L^{2}\left(0, T ; H^{-1}(\Omega)\right)$ і $A(x) \in L^{2}\left(\Omega ; \mathbb{S}^{N}\right)$ будемо називати функцію $y=y(x, t) \in L^{2}\left(0, T ; H_{0}^{1}(\Omega)\right)$, якщо має місце така інтегральна тотожність

$$
\begin{aligned}
& \int_{0}^{T} \int_{\Omega} y_{t} \varphi d x d t+\int_{0}^{T} \int_{\Omega}(\nabla \varphi,\nabla y+A \nabla y)_{\mathbb{R}^{N}} d x d t= \\
&=\int_{0}^{T} \int_{\Omega} f \varphi d x d t+\int_{0}^{T} \int_{\Omega} u \varphi d \mathcal{H}^{N-1} d t
\end{aligned}
$$

для будь-якого $\varphi \in C^{\infty}\left(0, T ; C_{0}^{\infty}(\Omega)\right)$ і при цьому

$$
y\left(t_{0}, x\right)=y_{0} \in L^{2}(\Omega) \quad \text { на } \Omega .
$$

Питання про існування слабкого розв'язку крайової задачі (3.1)-(3.3) в сенсі наведеного означення буде розглянуто в наступному розділі.

Оскільки $A(x) \in L^{2}\left(\Omega ; \mathbb{S}^{N}\right)$, то введемо до розгляду таку множину.

Означення 3.2. Будемо казати, що елемент $y \in L^{2}\left(0, T ; H_{0}^{1}(\Omega)\right)$ належить до множини $D$, якщо

$$
\left|\int_{0}^{T} \int_{\Omega}(\nabla \varphi, A \nabla y)_{\mathbb{R}^{N}} d x d t\right| \leq c(y)\left(\int_{0}^{T} \int_{\Omega}|\nabla \varphi|^{2} d x d t\right)^{1 / 2}
$$

для всіх $\varphi \in C^{\infty}\left(0, T ; C_{0}^{\infty}(\Omega)\right)$, де $c(y)$ - стала, яка залежить від $y$.

Далі введемо до розгляду білінійну форму

$$
[y, \varphi]=\int_{0}^{T} \int_{\Omega}(\nabla \varphi, A \nabla y)_{\mathbb{R}^{N}} d x d t \forall y \in D, \forall \varphi \in C^{\infty}\left(0, T ; C_{0}^{\infty}(\Omega)\right)
$$

і означимо білінійну форму $[y, \varphi]$ для всіх $\varphi \in L^{2}\left(0, T ; H_{0}^{1}(\Omega)\right)$ за правилом

$$
[y, \varphi]=\lim _{\varepsilon \rightarrow \infty}\left[y, \varphi_{\varepsilon}\right]
$$

де $\left\{\varphi_{\varepsilon}\right\}_{\varepsilon>0} \subset C^{\infty}\left(0, T ; C_{0}^{\infty}(\Omega)\right)$ і $\varphi_{\varepsilon} \rightarrow \varphi$ сильно в $L^{2}\left(0, T ; H_{0}^{1}(\Omega)\right)$. 
Твердження 3.1. Нехай $u \in L^{2}\left(0, T ; L^{2}\left(\Gamma_{2}\right)\right)$ є довільним допустимим керуванням. Тоді $y \in D$ за умови, що $y \in L^{2}\left(0, T ; H_{0}^{1}(\Omega)\right)$ - слабкий розв'язок задачі (3.1)-(3.3) в сенсі означення 3.1.

Доведення. Спочатку перепишемо інтегральну тотожність (3.5) у вигляді:

$$
\begin{aligned}
{[y, \varphi]=-\int_{0}^{T} \int_{\Omega} y_{t} \varphi d x d t-} & \int_{0}^{T} \int_{\Omega}(\nabla \varphi, \nabla y)_{\mathbb{R}^{N}} d x d t+ \\
& +\int_{0}^{T} \int_{\Omega} f \varphi d x d t+\int_{0}^{T} \int_{\Omega} u \varphi d \mathcal{H}^{N-1} d t .
\end{aligned}
$$

Застосувавши до (3.7) нерівність Гельдера, теорему Соболева про сліди і врахувавши наявність вкладення $H^{\frac{1}{2}}\left(\Gamma_{2}\right) \hookrightarrow L^{2}\left(\Gamma_{2}\right) \hookrightarrow H^{-\frac{1}{2}}\left(\Gamma_{2}\right)$, отримаємо:

$$
\begin{aligned}
|[y, \varphi]| \leq & \left(\left\|y_{t}\right\|_{L^{2}\left(0, T ; H^{-1}(\Omega)\right)}+\|y\|_{L^{2}\left(0, T ; H_{0}^{1}(\Omega)\right)}+\right. \\
& \left.+\|f\|_{L^{2}\left(0, T ; H^{-1}(\Omega)\right)}+C(\Omega, n)\|u\|_{L^{2}\left(0, T ; L^{2}\left(\Gamma_{2}\right)\right)}\right)\|\varphi\|_{L^{2}\left(0, T ; H_{0}^{1}(\Omega)\right)} .
\end{aligned}
$$

Зауваження 3.1. В силу твердження 3.1, інтегральну тотожність (3.5) можна переписати таким чином: $y$ є слабким розв'язком задачі (3.1)-(3.3) тоді і тільки тоді, коли $y \in D$ і має місце рівність

$$
\begin{aligned}
\int_{0}^{T} \int_{\Omega} & (\nabla \varphi, \nabla y)_{\mathbb{R}^{N}} d x d t+[y, \varphi]=-\int_{0}^{T} \int_{\Omega} y_{t} \varphi d x d t+ \\
& +\int_{0}^{T} \int_{\Omega} f \varphi d x d t+\int_{0}^{T} \int_{\Omega} u \varphi d \mathcal{H}^{N-1} d t \quad \forall \varphi \in L^{2}\left(0, T ; H_{0}^{1}(\Omega)\right) .
\end{aligned}
$$

Звідси випливає, що $y \in D$ - слабкий розв'язок задачі (3.1)-(3.3) і він задовольняє енергетичну рівність:

$$
\begin{aligned}
\|y\|_{L^{2}\left(0, T ; H_{0}^{1}(\Omega)\right)}^{2} & +[y, y]+\frac{1}{2}\|y(T)\|_{H_{0}^{1}(\Omega)}^{2}= \\
& =\int_{0}^{T} \int_{\Omega} f y d x d t+\int_{0}^{T} \int_{\Omega} u y d \mathcal{H}^{N-1} d t+\frac{1}{2}\left\|y_{0}\right\|_{H_{0}^{1}(\Omega)}^{2}
\end{aligned}
$$

У зв'язку з цим уведемо таке поняття.

Означення 3.3. Для задачі оптимального керування (3.1)-(3.4) пару $(u, y)$ будемо називати допустимою, якщо $u \in L^{2}\left(0, T ; L^{2}\left(\Gamma_{2}\right)\right), y \in D$ i пара $(u, y)$ задовольняє енергетичну рівність (3.10). Надалі $\Xi-$ множина всіх допустимих пар задачі (3.1)-(3.4). У свою чергу, пару $\left(u_{0}, y_{0}\right) \in L^{2}\left(0, T ; L^{2}\left(\Gamma_{2}\right)\right) \times D$ будемо називати оптимальною для задачі (3.1)-(3.4), якщо

$$
\left(u_{0}, y_{0}\right) \in \Xi \quad \text { i } I\left(u_{0}, y_{0}\right)=\inf _{(u, y) \in \Xi} I(u, y) .
$$


Теорема 3.1. Нехай для задачі (3.1)-(3.4) множина $\Xi$ н непорожнъою. Тодi для кожсного $f \in L^{2}\left(0, T ; H^{-1}(\Omega)\right)$ i $A(x) \in L^{2}\left(\Omega ; \mathbb{S}^{N}\right)$ існуе единий розв'язок $(u, y) \in L^{2}\left(0, T ; L^{2}\left(\Gamma_{2}\right)\right) \times D$ задачі (3.1)-(3.4).

Доведення. Оскільки $\Xi \neq \varnothing$ і функціонал (3.4) обмежений знизу на $\Xi$, існує мінімізаційна послідовність $\left\{\left(u^{k}, y^{k}\right)\right\}_{k \in \mathbb{N}} \subset \Xi$ для задачі (3.1)-(3.4) така, що

$$
\lim _{k \rightarrow \infty} I\left(u_{k}, y_{k}\right)=\inf _{(u, y) \in \Xi} I(u, y) \geq 0 .
$$

Таким чином, $\sup _{k \in \mathbb{N}} I\left(u^{k}, y^{k}\right) \leq C$ i

$$
\begin{aligned}
& \sup _{k \in \mathbb{N}}\left[\left\|y^{k}\right\|_{L^{2}\left(0, T ; H_{0}^{1}(\Omega)\right)}^{2}+\left\|u^{k}\right\|_{L^{2}\left(0, T ; L^{2}\left(\Gamma_{2}\right)\right)}^{2}\right] \leq \\
\leq & 2\left\|y_{d}\right\|_{L^{2}\left(0, T ; H_{0}^{1}(\Omega)\right)}^{2}+2 \sup _{k \in \mathbb{N}} I\left(u^{k}, y^{k}\right) \leq 2(1+C) .
\end{aligned}
$$

Звідси випливає, що існує підпослідовність $\left\{\left(u_{m}^{k}, y_{m}^{k}\right)\right\}_{m \in \mathbb{N}} \subset\left\{\left(u^{k}, y^{k}\right)\right\}_{k \in \mathbb{N}}$ така, що

$$
\begin{gathered}
u_{m}^{k} \rightarrow u_{0} \quad \text { в } L^{2}\left(0, T ; L^{2}\left(\Gamma_{2}\right)\right), \\
y_{m}^{k} \rightarrow y_{0} \quad \text { в } L^{2}\left(0, T ; H_{0}^{1}(\Omega)\right), \\
I\left(u_{0}, y_{0}\right)<+\infty .
\end{gathered}
$$

Оскільки

$$
\left[y^{k}, \varphi\right]=\int_{0}^{T} \int_{\Omega}\left(\nabla \varphi, A \nabla y^{k}\right)_{\mathbb{R}^{N}} d x d t=-\int_{0}^{T} \int_{\Omega}\left(A \nabla \varphi, \nabla y^{k}\right)_{\mathbb{R}^{N}} d x d t=\left[\varphi, y^{k}\right]
$$

i $A \varphi \in L^{2}\left(\Omega ; \mathbb{S}^{N}\right)$ для будь-якого $\varphi \in C^{\infty}\left(0, T ; C_{0}^{\infty}(\Omega)\right)$, можна перейти до границі в (3.5) при $u=u^{k}$ i $y=y^{k}$ коли $k \rightarrow \infty$. Таким чином, пара $\left(u_{0}, y_{0}\right)$ задовольняє (3.5), звідси $y_{0} \in D$ за твердженням 3.1. Отже, пара $\left(u_{0}, y_{0}\right) \in$ допустимою для задачі (3.1)-(3.4). Використовуючи властивість напівнеперервності знизу функціонала $I$ відносно добутку слабких топологій $L^{2}\left(\Gamma_{2}\right) \times H^{1}(\Omega)$, отримаємо:

$$
0 \leq I\left(u_{0}, y_{0}\right) \leq \lim _{k \rightarrow \infty} I\left(u_{k}, y_{k}\right)=I_{\min } .
$$

Таким чином, пара $\left(u_{0}, y_{0}\right)$ є оптимальною для задачі $(3.1)-(3.4)$, єдиність якої випливає із строгої опуклості функціонала $I$ на

$$
L^{2}\left(0, T ; L^{2}\left(\Gamma_{2}\right)\right) \times L^{2}\left(0, T ; H_{0}^{1}(\Omega)\right) .
$$




\section{4. Варіаційні розв'язки задачі оптимального керування}

Розглянемо умови, за яких поставлена задача оптимального керування має розв'язки.

Нехай $A \in L^{2}\left(\Omega ; \mathbb{S}^{N}\right)$. Тоді існує послідовність кососиметричних матриць $\left\{A_{k}\right\}_{k \in \mathbb{N}} \in L^{\infty}\left(\Omega ; \mathbb{S}^{N}\right)$ така, що

$$
A_{k} \rightarrow A \text { сильно в } L^{2}\left(\Omega ; \mathbb{S}^{N}\right) .
$$

Розглянемо послідовність апроксимованих задач оптимального керування, пов'язаних із матрицями $A_{k}$ :

$$
\begin{gathered}
y_{t}-\operatorname{div}\left(\nabla y+A_{k} \nabla y\right)=f \quad \text { на } \Omega \times[0, T], \\
y=0 \quad \text { на } \quad \Gamma_{1} \times[0, T], \quad \partial y / \partial \nu_{A_{k}}=u \quad \text { на } \Gamma_{2} \times[0, T], \\
y\left(t_{0}, x\right)=y_{0} \in L^{2}(\Omega) \quad \text { на } \Omega, \\
I_{k}(u, y):=I(u, y) \rightarrow \inf \\
\forall(u, y) \in L^{2}\left(0, T ; L^{2}\left(\Gamma_{2}\right)\right) \times L^{2}\left(0, T ; H_{0}^{1}(\Omega)\right) .
\end{gathered}
$$

Для подальшого розгляду задач оптимального керування (4.1)-(4.4) спочатку отримаємо оцінки для розв'язків задач (4.1)-(4.3), застосувавши метод Гальоркіна. Для цього введемо послідовність гладких функцій $\left\{w_{k}\right\}_{k=1}^{\infty}$ таких, що в просторі $H_{0}^{1}(\Omega)$ вони утворюють ортогональний базис, а в просторі $L^{2}(\Omega)$ - ортонормований. Далі побудуємо послідовність скінченно-вимірних підпросторів $V_{m}=\operatorname{span}\left\{w_{1}, \ldots, w_{m}\right\}$ таких, що

$$
V_{m} \subset V_{m+1} \quad \text { i } \quad \overline{\cup V_{m}}=H_{0}^{1}(\Omega) .
$$

Для фіксованого $m$ покладемо:

$$
y_{m}(t)=\sum_{k=1}^{m} c_{k}(t) w_{k} \quad \text { i } \quad G_{m}=\sum_{k=1}^{m} y_{0 k}(t) w_{k} .
$$

Тепер розглянемо наступну наближену задачу для $y_{m} \in H^{1}\left(0, T ; H_{0}^{1}(\Omega)\right), \forall s=$ $\overline{1, m}$

$$
\left\{\begin{array}{l}
\int_{0}^{T}\left(\dot{y}_{m}, w_{s}\right)_{H_{0}^{1}(\Omega)} d t+\int_{0}^{T} \int_{\Omega}\left(\nabla w_{s}, \nabla y_{m}+A_{k} \nabla y_{m}\right)_{\mathbb{R}^{N}} d x d t= \\
=\int_{0}^{T} \int_{\Omega} f w_{s} d x d t+\int_{0}^{T} \int_{\Omega} u w_{s} d \mathcal{H}^{N-1} d t \\
y_{m}(0)=G_{m}
\end{array}\right.
$$

Для цієї задачі має місце такий результат:

Лема 4.1. [1] Для будъ-якого $m$ існуе единий розв'язок $y_{m} \in H^{1}\left(0, T ; V_{m}\right)$ задачі (4.5). При ивому $y_{m} \in C\left([0, T] ; V_{m}\right)$. 
Тепер покажемо, що послідовності $\left\{y_{m}\right\}$ і $\left\{\dot{y}_{m}\right\}$ обмежені в $L^{2}\left(0, T ; H_{0}^{1}(\Omega)\right)$ і в $L^{2}\left(0, T ; H^{-1}(\Omega)\right)$ відповідно.

Теорема 4.1. Нехай $y_{m}$ единий розв'язок задачі (4.5), тоді для $\forall t \in[0, T]$ має місце така оцінка:

$$
\begin{aligned}
&\left\|y_{m}\right\|_{L^{2}\left(0, T ; H_{0}^{1}(\Omega)\right)}^{2}+\frac{1}{2}\left\|y_{m}(T)\right\|_{H_{0}^{1}(\Omega)}^{2} \leq\left(C_{1}\|u\|_{L^{2}\left(0, T ; L^{2}(\Omega)\right)}+\right. \\
&\left.+C\|f\|_{L^{2}\left(0, T ; H^{-1}(\Omega)\right)}+\frac{1}{\sqrt{2}}\left\|y_{0}\right\|_{H_{0}^{1}(\Omega)}\right)\left(C_{1}\|u\|_{L^{2}\left(0, T ; L^{2}(\Omega)\right)}+\right. \\
&\left.\quad+C\|f\|_{L^{2}\left(0, T ; H^{-1}(\Omega)\right)}\right)+\frac{1}{2}\left\|y_{0}\right\|_{H_{0}^{1}(\Omega)}^{2}
\end{aligned}
$$

Доведення. Помножимо перше рівняння (4.5) на $c_{k}(t)$ і просумуємо для $k=$ $\overline{1, m}$. В результаті отримаємо:

$$
\begin{aligned}
& \int_{0}^{T} \int_{\Omega} \dot{y}_{m} y_{m} d x d t+\int_{0}^{T} \int_{\Omega}\left(\nabla y_{m}, \nabla y_{m}+A_{k} \nabla y_{m}\right)_{\mathbb{R}^{N}} d x d t= \\
&=\int_{0}^{T} \int_{\Omega} f y_{m} d x d t+\int_{0}^{T} \int_{\Omega} u y_{m} d \mathcal{H}^{N-1} d t .
\end{aligned}
$$

Застосувавши Remark 7.10 з [1], теорему про сліди в просторах Соболєва і врахувавши те, що $\left\|G_{m}\right\|_{H_{0}^{1}(\Omega)}^{2} \leq\left\|y_{0}\right\|_{H_{0}^{1}(\Omega)}^{2} \mathrm{i}$

$$
\int_{0}^{T} \int_{\Omega}\left(\nabla y_{m}, A_{k} \nabla y_{m}\right)_{\mathbb{R}^{N}} d x d t=0
$$

отримаємо:

$$
\begin{aligned}
\left\|y_{m}\right\|_{L^{2}\left(0, T ; H_{0}^{1}(\Omega)\right)}^{2}+\frac{1}{2}\left\|y_{m}(T)\right\|_{H_{0}^{1}(\Omega)}^{2} & \leq\left\|y_{m}\right\|_{L^{2}\left(0, T ; H_{0}^{1}(\Omega)\right)} \\
\left(C_{1}\|u\|_{L^{2}\left(0, T ; L^{2}(\Omega)\right)}\right. & \left.+C\|f\|_{L^{2}\left(0, T ; H^{-1}(\Omega)\right)}\right)+\frac{1}{2}\left\|y_{0}\right\|_{H_{0}^{1}(\Omega)} .
\end{aligned}
$$

Звідки:

$$
\begin{aligned}
&\left\|y_{m}\right\|_{L^{2}\left(0, T ; H_{0}^{1}(\Omega)\right)}^{2}+\frac{1}{2}\left\|y_{m}(T)\right\|_{H_{0}^{1}(\Omega)}^{2} \leq\left(C_{1}\|u\|_{L^{2}\left(0, T ; L^{2}(\Omega)\right)}+\right. \\
&\left.+C\|f\|_{L^{2}\left(0, T ; H^{-1}(\Omega)\right)}+\frac{1}{\sqrt{2}}\left\|y_{0}\right\|_{H_{0}^{1}(\Omega)}\right)\left(C_{1}\|u\|_{L^{2}\left(0, T ; L^{2}(\Omega)\right)}+\right. \\
&\left.+C\|f\|_{L^{2}\left(0, T ; H^{-1}(\Omega)\right)}\right)+\frac{1}{2}\left\|y_{0}\right\|_{H_{0}^{1}(\Omega)}^{2}
\end{aligned}
$$

Теорема 4.2. Нехай $y_{m}-$ єдиний розв'язок задачі (4.5), тоді для $\forall t \in[0, T]$ мае місце така оцінка:

$$
\begin{aligned}
& \left\|\dot{y}_{m}\right\|_{L^{2}\left(0, T ; H^{-1}(\Omega)\right)}^{2} \leq 2\left\|y_{m}\right\|_{L^{2}\left(0, T ; H_{0}^{1}(\Omega)\right)}^{2}+2 C_{p}^{2}\|f\|_{L^{2}\left(0, T ; H^{-1}(\Omega)\right)}^{2}+ \\
& \quad+4 C_{p} C\|f\|_{L^{2}\left(0, T ; H^{-1}(\Omega)\right)}\|u\|_{L^{2}\left(0, T ; L^{2}(\Omega)\right)}+C^{2}\|u\|_{L^{2}\left(0, T ; L^{2}(\Omega)\right)}^{2} .
\end{aligned}
$$


Доведення. Нехай $v \in V$, тоді покладемо $v=w+z$, де $w \in V_{m} \mathrm{i} z \in V_{m}^{\perp}$. Отже, $\|\nabla w\|_{H_{0}^{1}(\Omega)} \leq\|\nabla v\|_{H_{0}^{1}(\Omega)}$. Слід зазначити, що рівняння з (4.5) має місце для кожного елемента базису $w_{s}$ для $s=\overline{1, m}$ тоді і тільки тоді, коли воно має місце для $\forall v \in V_{m}$. Тепер перепишемо (4.5):

$$
\left\{\begin{array}{l}
\int_{0}^{T} \int_{\Omega} \dot{y}_{m} v d x d t+\int_{0}^{T} \int_{\Omega}\left(\nabla v, \nabla y_{m}+A_{k} \nabla y_{m}\right)_{\mathbb{R}^{N}} d x d t= \\
=\int_{0}^{T} \int_{\Omega} f v d x d t+\int_{0}^{T} \int_{\Omega} u v d \mathcal{H}^{N-1} d t \\
y_{m}(0)=G_{m} .
\end{array}\right.
$$

Далі в задачі (4.11) покладемо $v=w$. В результаті отримаємо:

$$
\begin{aligned}
\int_{0}^{T} \int_{\Omega} \dot{y}_{m} w d x d t=-\int_{0}^{T} \int_{\Omega} & \left(\nabla w, \nabla y_{m}+A_{k} \nabla y_{m}\right)_{\mathbb{R}^{N}} d x d t+ \\
& +\int_{0}^{T} \int_{\Omega} f w d x d t+\int_{0}^{T} \int_{\Omega} u w d \mathcal{H}^{N-1} d t .
\end{aligned}
$$

Застосувавши нерівність Шварца - Пуанкаре, теорему про сліди в просторах Соболєва і врахувавши, що

$$
\int_{\Omega} \dot{y}_{m} w d x=\int_{\Omega} \dot{y}_{m} v d x, \quad \int_{0}^{T} \int_{\Omega}\left(\nabla w, A_{k} \nabla y_{m}\right)_{\mathbb{R}^{N}} d x d t
$$

отримаємо:

$$
\begin{gathered}
\left\|\dot{y_{m}}\right\|_{H^{-1}(\Omega)}\|v\|_{H_{0}^{1}(\Omega)} \leq\left\|y_{m}\right\|_{H_{0}^{1}(\Omega)}\|w\|_{H_{0}^{1}(\Omega)}+C_{p}\|f\|_{H^{-1}(\Omega)}\|w\|_{H_{0}^{1}(\Omega)}+ \\
+C\|u\|_{L^{2}\left(\Gamma_{2}\right)}\|w\|_{H_{0}^{1}(\Omega)} \leq\left\|y_{m}\right\|_{H_{0}^{1}(\Omega)}\|v\|_{H_{0}^{1}(\Omega)}+ \\
+C_{p}\|f\|_{H^{-1}(\Omega)}\|v\|_{H_{0}^{1}(\Omega)}+C\|u\|_{L^{2}\left(\Gamma_{2}\right)}\|v\|_{H_{0}^{1}(\Omega)}
\end{gathered}
$$

Тепер застосуємо нерівність $(a+b)^{2} \leq 2 a^{2}+2 b^{2}$ і проінтегруємо отриманий результат відносно $t$ на інтервалі $(0, T)$ :

$$
\begin{aligned}
& \left\|\dot{y}_{m}\right\|_{L^{2}\left(0, T ; H^{-1}(\Omega)\right)}^{2} \leq 2\left\|y_{m}\right\|_{L^{2}\left(0, T ; H_{0}^{1}(\Omega)\right)}^{2}+2 C_{p}^{2}\|f\|_{L^{2}\left(0, T ; H^{-1}(\Omega)\right)}^{2}+ \\
& \quad+4 C_{p} C\|f\|_{L^{2}\left(0, T ; H^{-1}(\Omega)\right)}\|u\|_{L^{2}\left(0, T ; L^{2}(\Omega)\right)}+C^{2}\|u\|_{L^{2}\left(0, T ; L^{2}(\Omega)\right)}^{2}
\end{aligned}
$$

Таким чином, теореми 4.1 і 4.2 показують, що послідовності наближень Гальоркіна $\left\{y_{m}\right\} \subset L^{2}\left(0, T ; H_{0}^{1}(\Omega)\right)$ i $\left\{\dot{y}_{m}\right\} \subset L^{2}\left(0, T ; H^{-1}(\Omega)\right)$ обмежені у відповідних просторах, а отже, за теоремою Банаха - Алаоглу можна вилучити підпослідовності $\left\{y_{m_{n}}\right\}$ i $\left\{\dot{y}_{m_{n}}\right\}$ для $n \rightarrow \infty$ такі, що

$$
\begin{gathered}
y_{m_{n}} \rightarrow y^{k} \quad \text { слабо в } \quad L^{2}\left(0, T ; H_{0}^{1}(\Omega)\right), \\
\dot{y}_{m_{n}} \rightarrow \dot{y}^{k} \quad \text { слабо в } \quad L^{2}\left(0, T ; H^{-1}(\Omega)\right),
\end{gathered}
$$

де $y^{k}-$ розв'язок задачі $(4.1)-(4.3)$, для якого має місце результат. 
Теорема 4.3. [1] Нехай $u \in L^{2}\left(0, T ; L^{2}\left(\Gamma_{2}\right)\right), f \in L^{2}\left(0, T ; H^{-1}(\Omega)\right)$. Тоді для $\forall A_{k} \in L^{\infty}\left(\Omega ; \mathbb{S}^{N}\right)$ елемент $y^{k} \in L^{2}\left(0, T ; H_{0}^{1}(\Omega)\right)$ - единий розв'язок задачі (4.1)-(4.3). Крім того, мають місце оцінки:

$$
\begin{gathered}
\left\|y^{k}\right\|_{L^{2}\left(0, T ; H_{0}^{1}(\Omega)\right)}^{2}+\frac{1}{2}\left\|y^{k}(T)\right\|_{H_{0}^{1}(\Omega)}^{2} \leq\left(C_{1}\|u\|_{L^{2}\left(0, T ; L^{2}(\Omega)\right)}+\right. \\
\left.+C\|f\|_{L^{2}\left(0, T ; H^{-1}(\Omega)\right)}+\frac{1}{\sqrt{2}}\left\|y_{0}\right\|_{H_{0}^{1}(\Omega)}\right)\left(C_{1}\|u\|_{L^{2}\left(0, T ; L^{2}(\Omega)\right)}+\right. \\
\left.+C\|f\|_{L^{2}\left(0, T ; H^{-1}(\Omega)\right)}\right)+\frac{1}{2}\left\|y_{0}\right\|_{H_{0}^{1}(\Omega)}^{2}, \\
\left\|\dot{y}^{k}\right\|_{L^{2}\left(0, T ; H^{-1}(\Omega)\right)}^{2} \leq 2\left\|y^{k}\right\|_{L^{2}\left(0, T ; H_{0}^{1}(\Omega)\right)}^{2}+2 C_{p}^{2}\|f\|_{L^{2}\left(0, T ; H^{-1}(\Omega)\right)}^{2}+ \\
\quad+4 C_{p} C\|f\|_{L^{2}\left(0, T ; H^{-1}(\Omega)\right)}\|u\|_{L^{2}\left(0, T ; L^{2}(\Omega)\right)}+C^{2}\|u\|_{L^{2}\left(0, T ; L^{2}(\Omega)\right)}^{2}
\end{gathered}
$$

Як наслідок отримаємо результат, який стосується слабкого розв'язку задачі (3.1)-(3.3)

Твердження 4.1. Нехай $u \in L^{2}\left(0, T ; L^{2}\left(\Gamma_{2}\right)\right), f \in L^{2}\left(0, T ; H^{-1}(\Omega)\right)$ i $A \in$ $L^{2}\left(\Omega ; \mathbb{S}^{N}\right)$. Нехай для $\forall k \in \mathbb{N} y^{k}-$ відповідні розв'язки задач (4.1)-(4.3). Тоді:

(i) $y^{k} \rightarrow y^{*} \quad$ слабо в $L^{2}\left(0, T ; H_{0}^{1}(\Omega)\right)$,

(ii) $\dot{y}^{k} \rightarrow \dot{y}^{*} \quad$ слабо в $L^{2}\left(0, T ; H^{-1}(\Omega)\right)$,

де $y^{*}$ - слабкий розв'язок задачі (3.1)-(3.3). Крім того, має місце нерівність:

$$
\left[y^{*}, y^{*}\right] \geq 0 \text {. }
$$

Доведення. Оскільки $y^{k}-$ слабкий розв' язок задачі (4.1)-(4.3), то має місце інтегральна тотожність

$$
\begin{aligned}
& \int_{0}^{T} \int_{\Omega} y_{t}^{k} \varphi d x d t+\int_{0}^{T} \int_{\Omega}(\nabla \varphi\left., \nabla y^{k}+A_{k} \nabla y^{k}\right)_{\mathbb{R}^{N}} d x d t= \\
&=\int_{0}^{T} \int_{\Omega} f \varphi d x d t+\int_{0}^{T} \int_{\Omega} u \varphi d \mathcal{H}^{N-1} d t
\end{aligned}
$$

для будь-якого $\varphi \in C^{\infty}\left(0, T ; C_{0}^{\infty}(\Omega)\right)$. Враховуючи той факт, що

$$
\int_{\Omega}\left(\nabla v, \nabla v+A_{k} \nabla v\right)_{\mathbb{R}^{N}} d x=0 \quad \forall v \in H_{0}^{1}(\Omega),
$$

отримаємо:

$$
\begin{aligned}
\int_{0}^{T} \int_{\Omega} y_{t}^{k} y^{k} d x d t+\int_{0}^{T} \int_{\Omega} & \left(\nabla y^{k}, \nabla y^{k}\right)_{\mathbb{R}^{N}} d x d t= \\
=\int_{0}^{T} \int_{\Omega} f y^{k} d x d t & +\int_{0}^{T} \int_{\Omega} u y^{k} d \mathcal{H}^{N-1} d t
\end{aligned}
$$


або

$$
\begin{aligned}
\left\|y^{k}\right\|_{L^{2}\left(0, T ; H_{0}^{1}(\Omega)\right)}^{2}+\frac{1}{2}\left\|y^{k}(T)\right\|_{H_{0}^{1}(\Omega)}^{2} & =\int_{0}^{T} \int_{\Omega} f y^{k} d x d t+ \\
& +\int_{0}^{T} \int_{\Omega} u y^{k} d \mathcal{H}^{N-1} d t+\frac{1}{2}\left\|y_{0}(T)\right\|_{H_{0}^{1}(\Omega)}^{2}
\end{aligned}
$$

Із теореми 4.3 випливає, що $\left\{y^{k}\right\}$ і $\left\{\dot{y}^{k}\right\}$ обмежені в просторах $L^{2}\left(0, T ; H_{0}^{1}(\Omega)\right)$ і $L^{2}\left(0, T ; H^{-1}(\Omega)\right)$ відповідно. Отже, існує елемент $y^{*} \in L^{2}\left(0, T ; H_{0}^{1}(\Omega)\right)$ такий, що

$$
\begin{array}{ccc}
y^{k} \rightarrow y^{*} & \text { слабо в } & L^{2}\left(0, T ; H_{0}^{1}(\Omega)\right), \\
\dot{y}^{k} \rightarrow \dot{y}^{*} \quad \text { слабо в } & L^{2}\left(0, T ; H^{-1}(\Omega)\right) .
\end{array}
$$

Таким чином,

$$
\int_{0}^{T} \int_{\Omega}\left(\nabla \varphi, \nabla y^{k}\right)_{\mathbb{R}^{N}} d x d t \rightarrow \int_{0}^{T} \int_{\Omega}\left(\nabla \varphi, \nabla y^{*}\right)_{\mathbb{R}^{N}} d x d t
$$

i

$$
\int_{0}^{T} \int_{\Omega} y_{t}^{k} \varphi d x d t \rightarrow \int_{0}^{T} \int_{\Omega} y_{t}^{*} \varphi d x d t
$$

для будь-якого $\varphi \in C^{\infty}\left(0, T ; C_{0}^{\infty}(\Omega)\right)$. Далі розглянемо:

$$
\begin{gathered}
\int_{0}^{T} \int_{\Omega}\left(\nabla \varphi, A \nabla y^{*}-A_{k} \nabla y^{k}\right)_{\mathbb{R}^{N}} d x d t \leq \mid \int_{0}^{T} \int_{\Omega}\left(\nabla \varphi, A \nabla y^{*} \pm A \nabla y^{k}-\right. \\
\left.-A_{k} \nabla y^{k}\right)_{\mathbb{R}^{N}} d x d t|\leq| \int_{0}^{T} \int_{\Omega}\left(\nabla \varphi, A \nabla y^{*}-A \nabla y^{k}\right)_{\mathbb{R}^{N}} d x d t \mid+ \\
+\left|\int_{0}^{T} \int_{\Omega}\left(\nabla \varphi, A \nabla y^{k}-A_{k} \nabla y^{k}\right)_{\mathbb{R}^{N}} d x d t\right| \leq\left|\int_{0}^{T} \int_{\Omega}\left(A \nabla \varphi, \nabla y^{*}-\nabla y^{k}\right)_{\mathbb{R}^{N}} d x d t\right|+ \\
+\left\|A-A_{k}\right\|_{L^{2}\left(\Omega ; \mathbb{S}^{N}\right)} \int_{0}^{T}\left\|y^{k}\right\|_{H_{0}^{1}(\Omega)}\|\varphi\|_{C_{0}^{\infty}(\Omega)} d t \leq \\
\leq\left|\int_{0}^{T} \int_{\Omega}\left(A \nabla \varphi, \nabla y^{*}\right)_{\mathbb{R}^{N}} d x d t-\int_{0}^{T} \int_{\Omega}\left(A \nabla \varphi, \nabla y^{k}\right)_{\mathbb{R}^{N}} d x d t\right|+ \\
+\left\|A-A_{k}\right\|_{L^{2}\left(\Omega ; \mathbb{S}^{N}\right)} \sup _{k \in \mathbb{N}}\left\|y^{k}\right\|_{L^{2}\left(0, T ; H_{0}^{1}(\Omega)\right)}\|\varphi\|_{C^{\infty}\left(0, T ; C_{0}^{\infty}(\Omega)\right)} \rightarrow 0 . \quad \text { (4.20) }
\end{gathered}
$$

Таким чином $A_{k} \nabla y^{k} \stackrel{*}{\rightarrow} A \nabla y^{*} \quad{ }^{*}$-слабо в $L^{1}\left(\Omega ; \mathbb{R}^{N}\right)$, а отже, можна перейти до границі в (4.17). В результаті отримаємо:

$$
\begin{aligned}
& \int_{0}^{T} \int_{\Omega} y_{t}^{*} \varphi d x d t+\int_{0}^{T} \int_{\Omega}\left(\nabla \varphi, \nabla y^{*}+A_{k} \nabla y^{*}\right)_{\mathbb{R}^{N}} d x d t= \\
&=\int_{0}^{T} \int_{\Omega} f \varphi d x d t+\int_{0}^{T} \int_{\Omega} u \varphi d \mathcal{H}^{N-1} d t .
\end{aligned}
$$


Звідси випливає, що $y^{*}-$ слабкий розв'язок задачі $(3.1)-(3.3)$ і $y^{*} \in D$. Оскільки $\nabla y^{k} \rightarrow \nabla y^{*}$ в $L^{2}(\Omega)$, то перейшовши до границі в (4.18) і застосувавши властивість напівнеперервності знизу норми $\|\cdot\|_{H^{1}(\Omega)}^{2}$, отримаємо:

$$
\begin{aligned}
\left\|y^{*}\right\|_{L^{2}\left(0, T ; H_{0}^{1}(\Omega)\right)}^{2} & +\frac{1}{2}\left\|y^{*}(T)\right\|_{H_{0}^{1}(\Omega)}^{2} \leq \\
& \leq \int_{0}^{T} \int_{\Omega} f y^{*} d x d t+\int_{0}^{T} \int_{\Omega} u y^{*} d \mathcal{H}^{N-1} d t+\frac{1}{2}\left\|y_{0}\right\|_{H_{0}^{1}(\Omega)}^{2} .
\end{aligned}
$$

Оскільки $y^{*}-$ слабкий розв'язок задачі (3.1)-(3.3), то $y^{*}$ задовольняє (3.10), а отже, з (4.22) випливає, що $\left[y^{*}, y^{*}\right] \geq 0$.

Тепер розглянемо задачу оптимального керування (4.1)-(4.4).

Твердження 4.2. Нехай $y_{d} \in L^{2}\left(0, T ; H_{0}^{1}(\Omega)\right), f \in L^{2}\left(0, T ; H^{-1}(\Omega)\right)$ і $A \in$ $L^{2}\left(\Omega ; \mathbb{S}^{N}\right)$ задано. Тоді для $\forall k \in \mathbb{N}$ існує єдина оптимальна пара $\left(u_{0}^{k}, y_{0}^{k}\right) \in$ $\Xi_{k}$ для відповідної задачі (4.1)-(4.3) така, що послідовність пар $\left\{\left(u_{0}^{k}, y_{0}^{k}\right) \in\right.$ $\left.\Xi_{k}\right\}_{k \in \mathbb{N}}$ відносно компактна в добутку слабких топологій на $L^{2}\left(\Gamma_{2}\right) \times H_{0}^{1}(\Omega)$, тобто

$$
y_{0}^{k} \rightarrow y^{*} \quad \text { слабо в } \quad L^{2}\left(0, T ; H_{0}^{1}(\Omega)\right), u_{0}^{k} \rightarrow u^{*} \quad \text { слабо в } \quad L^{2}\left(0, T ; L^{2}\left(\Gamma_{2}\right)\right),
$$

де $\left(u^{*}, y^{*}\right) \in \Xi$.

Доведення. Спочатку покажемо, що для послідовності задач (4.4) виконується умова $\sup _{k \in \mathbb{N}} \inf _{(u, y) \in \Xi} I_{k}(u, y) \leq C$. Оскільки для кожного $k \in \mathbb{N}$ білінійна форма $[y, \varphi]_{k}$ обмежена в $L^{2}\left(0, T ; H_{0}^{1}(\Omega)\right)$ і має місце рівність

$$
\int_{0}^{T} \int_{\Omega}\left(\nabla y^{k}, A \nabla y^{k}\right)_{\mathbb{R}^{N}} d x d t=0
$$

то за лемою Лакса - Мільграма задача (4.1)-(4.3) має єдиний розв'язок для кожного $y^{k} \in L^{2}\left(0, T ; H_{0}^{1}(\Omega)\right)$. Враховуючи те, що функціонал $I_{k}$ строго опуклий, і застосувавши властивість напівнеперервності, отримаємо, що задача (4.1)-(4.4) допускає єдиний розв'язок

$$
I_{k}\left(u_{0}^{k}, y_{0}^{k}\right)=\inf _{(u, y) \in \Xi} I_{k}(u, y), \quad\left(u_{0}^{k}, y_{0}^{k}\right) \in \Xi .
$$

Із теореми (4.3) випливає, що послідовність $\left\{y^{k}\right\}_{k \in \mathbb{N}} \in L^{2}\left(0, T ; H_{0}^{1}(\Omega)\right)$ для фіксованого $u \in L^{2}\left(0, T ; L^{2}\left(\Gamma_{2}\right)\right)$ буде обмеженою і має місце оцінка (4.15), де сталі $C$ і $C_{1}$ не залежать від $A_{k}$. В результаті:

$$
\begin{aligned}
& I_{k}\left(u_{0}^{k}, y_{0}^{k}\right)=\inf _{(u, y) \in \Xi} I_{k}(u, y) \leq I_{k}\left(u, y^{k}\right) \leq 2\left\|y_{d}\right\|_{L^{2}\left(0, T ; H_{0}^{1}(\Omega)\right)}+ \\
&+2\left(C_{1}\|u\|_{L^{2}\left(0, T ; L^{2}(\Omega)\right)}+C\|f\|_{L^{2}\left(0, T ; H^{-1}(\Omega)\right)}+\frac{1}{\sqrt{2}}\left\|y_{0}\right\|_{H_{0}^{1}(\Omega)}\right) \\
& \quad\left(C_{1}\|u\|_{L^{2}\left(0, T ; L^{2}(\Omega)\right)}+C\|f\|_{L^{2}\left(0, T ; H^{-1}(\Omega)\right)}\right)+\left\|y_{0}\right\|_{H_{0}^{1}(\Omega)}^{2}
\end{aligned}
$$


Таким чином,

$$
\sup _{k \in \mathbb{N}}\left(\left\|y_{0}^{k}\right\|_{L^{2}\left(0, T ; H_{0}^{1}(\Omega)\right)}^{2}+\left\|u_{0}^{k}\right\|_{L^{2}\left(0, T ; L^{2}(\Omega)\right)}\right)<+\infty,
$$

а, отже, послідовність пар $\left\{\left(u_{0}^{k}, y_{0}^{k}\right)\right\}_{k \in \mathbb{N}}$ слабо збігається, тобто

$$
y_{0}^{k} \rightarrow y^{*} \text { в } L^{2}\left(0, T ; H_{0}^{1}(\Omega)\right) \text { і } u_{0}^{k} \rightarrow u^{*} \text { в } L^{2}\left(0, T ; L^{2}\left(\Gamma_{2}\right)\right) .
$$

Урахувавши результати, отримані у твердженні 4.1, і перейшовши до границі в (4.18) з $u=u_{0}^{k}$, доходимо висновку, що пара $\left(u^{*}, y^{*}\right)$ пов'язана інтегральною тотожністю (3.5), де $y^{*}-$ слабкий розв'язок задачі $(3.1)-(3.3)$ для $u=u^{*}$ i $y^{*} \in D$. Отже, $\left(u^{*}, y^{*}\right) \in \Xi$.

Із наведених результатів випливає, що для будь-якого наближення $\left\{A_{k}\right\}_{k \in \mathbb{N}}$ $\in L^{\infty}\left(\Omega ; \mathbb{S}^{N}\right)$ матриці $A \in L^{2}\left(\Omega ; \mathbb{S}^{N}\right)$ з властивістю $A_{k} \rightarrow A$ в $L^{2}\left(\Omega ; \mathbb{S}^{N}\right)$, оптимальний розв' язок задачі оптимального керування (4.1)-(4.4) завжди дає в границі деякий допустимий, проте не обов'язково оптимальний, розв'язок $\left(u^{*}, y^{*}\right)$ задачі оптимального керування (3.1)-(3.4), який може залежати від вибору $\left\{A_{k}\right\}_{k \in \mathbb{N}}$. Таким чином, виникає питання щодо досяжності оптимальних розв'язків задачі (3.1)-(3.4) і структури множини всіх досяжних розв'язків. У зв'язку з цим далі наведемо поняття і результати, які стосуються умов досяжності оптимальних розв'язків задачі (3.1)-(3.4).

Зауваэсення 4.1. Надалі наближення $\left\{A_{k}\right\}_{k \in \mathbb{N}} \in L^{\infty}\left(\Omega ; \mathbb{S}^{N}\right)$ матриці $A \in$ $L^{2}\left(\Omega ; \mathbb{S}^{N}\right)$ з властивістю $A_{k} \rightarrow A$ сильно в $L^{2}\left(\Omega ; \mathbb{S}^{N}\right)$ будемо називати $L^{\infty}$ апроксимацією матриці $A$.

Означення 4.1. Пару $\left(u^{*}, y^{*}\right) \in L^{2}\left(0, T ; L^{2}\left(\Gamma_{2}\right)\right) \times L^{2}\left(0, T ; H_{0}^{1}(\Omega)\right)$ називатимемо варіаційним розв'язком задачі оптимального керування $(3.1)-(3.4)$, якщо існує $L^{\infty}$-апроксимація матриці $A$ така, що

$$
I\left(u^{*}, y^{*}\right)=\inf _{(u, y) \in \Xi} I(u, y), \quad\left(u^{*}, y^{*}\right) \in \Xi
$$

де $y_{0}^{k} \rightarrow y^{*}$ в $L^{2}\left(0, T ; H_{0}^{1}(\Omega)\right)$ і $u_{0}^{k} \rightarrow u^{*}$ в $L^{2}\left(0, T ; L^{2}\left(\Gamma_{2}\right)\right)$, i

$$
\left\langle\inf _{(u, y) \in \Xi_{k}} I_{k}(u, y)\right\rangle \stackrel{\text { Var }}{\longrightarrow}\left\langle\inf _{(u, y) \in \Xi} I(u, y)\right\rangle \text { при } k \rightarrow \infty .
$$

Твердження 4.3. Нехай $\left(u^{*}, y^{*}\right) \in L^{2}\left(0, T ; L^{2}\left(\Gamma_{2}\right)\right) \times L^{2}\left(0, T ; H_{0}^{1}(\Omega)\right)$ - варіаційний розв'язок задачі оптимального керування (3.1)-(3.4). Тоді $\left[y^{*}, y^{*}\right]=0$.

Доведення. Із теореми 2.1 і апріорних оцінок (4.15), (4.23) випливає, що

$$
y_{0}^{k} \rightarrow y^{*} \text { в } L^{2}\left(0, T ; H_{0}^{1}(\Omega)\right) \text { і } u_{0}^{k} \rightarrow u^{*} \text { в } L^{2}\left(0, T ; L^{2}\left(\Gamma_{2}\right)\right),
$$




$$
\begin{gathered}
\inf _{(u, y) \in \Xi} I(u, y)=I\left(u^{*}, y^{*}\right):=\left\|y^{*}-y_{d}\right\|_{L^{2}\left(0, T ; H_{0}^{1}(\Omega)\right)}^{2}+\left\|u^{*}\right\|_{L^{2}\left(0, T ; L^{2}(\Omega)\right)}^{2}= \\
=\lim _{k \rightarrow \infty} \inf _{\left(u^{k}, y^{k}\right) \in \Xi_{k}} I_{k}\left(u^{k}, y^{k}\right)=\lim _{k \rightarrow \infty} I_{k}\left(u_{0}^{k}, y_{0}^{k}\right)= \\
\quad=\lim _{k \rightarrow \infty}\left[\left\|y_{0}^{k}-y_{d}\right\|_{L^{2}\left(0, T ; H_{0}^{1}(\Omega)\right)}^{2}+\left\|u_{0}^{k}\right\|_{L^{2}\left(0, T ; L^{2}(\Omega)\right)}^{2}\right] .
\end{gathered}
$$

Таким чином, (4.15) є наслідком (4.26) і (4.27). Урахувавши той факт, що

$$
\int_{0}^{T} \int_{\Omega}\left(\nabla y_{0}^{k}, A \nabla y_{0}^{k}\right)_{\mathbb{R}^{N}} d x d t=0
$$

отримаємо:

$$
\begin{aligned}
& 0= \lim _{k \rightarrow \infty}\left[y_{0}^{k}, y_{0}^{k}\right]=-\lim _{k \rightarrow \infty}\left\|y_{0}^{k}\right\|_{L^{2}\left(0, T ; H_{0}^{1}(\Omega)\right)}^{2}-\frac{1}{2} \lim _{k \rightarrow \infty}\left\|y_{0}^{k}(T)\right\|_{H_{0}^{1}(\Omega)}^{2}+ \\
&+ \lim _{k \rightarrow \infty} \int_{0}^{T} \int_{\Omega} f y_{0}^{k} d x d t+\lim _{k \rightarrow \infty} \int_{0}^{T} \int_{\Omega} u y_{0}^{k} d \mathcal{H}^{N-1} d t+\frac{1}{2}\left\|y_{0}(T)\right\|_{H_{0}^{1}(\Omega)}^{2}= \\
&=-\left\|y^{*}\right\|_{L^{2}\left(0, T ; H_{0}^{1}(\Omega)\right)}^{2}-\frac{1}{2}\left\|y^{*}(T)\right\|_{H_{0}^{1}(\Omega)}^{2}+ \\
&+\int_{0}^{T} \int_{\Omega} f y^{*} d x d t+\int_{0}^{T} \int_{\Omega} u y^{*} d \mathcal{H}^{N-1} d t+\frac{1}{2}\left\|y_{0}\right\|_{H_{0}^{1}(\Omega)}^{2}=\left[y^{*}, y^{*}\right] .
\end{aligned}
$$

Тепер наведемо умови, які гарантують існування варіаційного розв'язку задачі оптимального керування (3.1)-(3.4).

Теорема 4.4. Нехай матриц,я $A \in L^{2}\left(\Omega ; \mathbb{S}^{N}\right)$ е такою, що

$$
[y, y]=0 \quad \forall y \in D
$$

Тоді єдиний розв'язок $\left(u_{0}, y_{0}\right)$ задачі оптималъного керування (3.1)-(3.4) е варіаційним.

Доведення. Нехай задано $L^{\infty}$-апроксимацію матриці $A \in L^{2}\left(\Omega ; \mathbb{S}^{N}\right)$. Далі розглянемо послідовність пар $\left\{\left(u^{k}, y^{k}\right)\right\}_{k \in \mathbb{N}} \subset L^{2}\left(0, T ; L^{2}\left(\Gamma_{2}\right)\right) \times L^{2}\left(0, T ; H_{0}^{1}(\Omega)\right)$, яка наділена такими властивостями:

(i) $\left\{\left(u^{k}, y^{k}\right)\right\}_{k \in \mathbb{N}} \in \Xi_{n_{k}}$, де $\left\{\left(n_{k}\right)\right\}_{k \in \mathbb{N}} \rightarrow \infty$ коли $k \rightarrow \infty$,

(ii) $y^{k} \rightarrow y$ в $L^{2}\left(0, T ; H_{0}^{1}(\Omega)\right)$ і $u^{k} \rightarrow u$ в $L^{2}\left(0, T ; L^{2}\left(\Gamma_{2}\right)\right)$.

Із твердження 4.2 випливає, що гранична пара $(u, y) \in$ допустимою для вихідної задачі (3.1)-(3.4), а отже, ця задача має єдиний оптимальний розв'язок $\left(u_{0}, y_{0}\right) \in \Xi$ за теоремою 3.1. Далі покажемо, що задача оптимального керування (3.1)-(3.4) є варіаційною границею послідовності задач (4.1)-(4.4). Для цього потрібно перевірити виконання умов означення 2.1 . 
Умова (а) є очевидною для простору $L^{2}\left(0, T ; L^{2}\left(\Gamma_{2}\right)\right) \times L^{2}\left(0, T ; H_{0}^{1}(\Omega)\right)$ для $\delta>0$. У свою чергу, умова (аа) випливає з нерівності

$$
\begin{gathered}
\lim \inf _{k \rightarrow \infty} I_{k}\left(u^{k}, y^{k}\right)= \\
=\lim \inf _{k \rightarrow \infty}\left[\left\|y^{k}-y_{d}\right\|_{L^{2}\left(0, T ; H_{0}^{1}(\Omega)\right)}^{2}+\left\|u^{k}\right\|_{L^{2}\left(0, T ; L^{2}(\Omega)\right)}^{2}\right] \geq I(u, y),
\end{gathered}
$$

яка має місце для будь-якої послідовності

$$
\left\{\left(u^{k}, y^{k}\right)\right\}_{k \in \mathbb{N}} \subset L^{2}\left(0, T ; L^{2}\left(\Gamma_{2}\right)\right) \times L^{2}\left(0, T ; H_{0}^{1}(\Omega)\right)
$$

із властивостями (i)-(ii).

Тепер перевіримо умову (ааа) означення 2.1. Отже, нехай $\left(u^{*}, y^{*}\right)$ довільна допустима пара вихідної задачі і нехай $\left\{\widehat{u}^{k}\right\}_{k \in \mathbb{N}} \in L^{2}\left(0, T ; L^{2}\left(\Gamma_{2}\right)\right)$ послідовність керувань таких, що

$$
\widehat{u}^{k} \rightarrow u^{*} \text { сильно в } L^{2}\left(0, T ; L^{2}\left(\Gamma_{2}\right)\right) .
$$

Нехай $\left\{\widehat{y}^{k}=\widehat{y}^{k}\left(u^{k}\right)\right\}_{k \in \mathbb{N}}$ відповідні розв'язки задачі оптимального керування (4.1)-(4.3). Тоді з твердження 4.2 випливає, що послідовність $\left\{\widehat{y}^{k}\right\}_{k \in \mathbb{N}}$ рівномірно обмежена в $L^{2}\left(0, T ; H_{0}^{1}(\Omega)\right)$ і існує елемент $\widehat{y} \in D$ такий, що $\left(u^{*}, \widehat{y}\right) \in \Xi$ i $\widehat{y}^{k} \rightarrow \widehat{y}$ в $L^{2}\left(0, T ; H_{0}^{1}(\Omega)\right)$. Тепер покажемо, що $\widehat{y}=y^{*}$ і має місце тотожність

$$
I\left(u^{*}, y^{*}\right)=\lim \sup _{k \rightarrow \infty} I_{k}\left(\widehat{u}^{k}, \widehat{y}^{k}\right) .
$$

Оскільки $\left(u^{*}, y^{*}\right) \in \Xi \mathrm{i}\left(u^{*}, \widehat{y}\right) \in \Xi$, то $y=y^{*}-\widehat{y}$ - розв'язок однорідної задачі

$$
\begin{gathered}
y_{t}-\operatorname{div}(\nabla y+A(x) \nabla y)=0 \quad \text { на } \Omega \times[0, T], \\
y=0 \quad \text { на } \quad \Gamma_{1} \times[0, T], \quad \partial y / \partial \nu_{A}=0 \quad \text { на } \Gamma_{2} \times[0, T], \\
y\left(t_{0}, x\right)=y_{0} \in L^{2}(\Omega) \text { на } \Omega .
\end{gathered}
$$

Слід зазначити, що ця задача має лише тривіальні розв'язки, оскільки

$$
\int_{0}^{T} \int_{\Omega} y_{t} y d x d t+\int_{0}^{T} \int_{\Omega}(\nabla y, \nabla y)_{\mathbb{R}^{N}} d x d t=-[y, y]=0 .
$$

Отже, $y^{*}=\widehat{y}$. Нерівність (4.31) випливає 3 (4.30) і з енергетичних оцінок (4.18), (3.10).

\section{Бібліографічні посилання}

1. Salsa S. Partial Differential Equations in Action: From Modelling to Theory / S. Salsa. - Milan : Springer-Verlag, 2008.

2. Zhikov $V$. $V$. Remarks on the uniqueness of a solution of the Dirichlet problem for second-order elliptic equations with lower-order terms / V. V. Zhikov - Functional Analysis and Its Applications, 38(2004), No. 3.- P. 173-183.

3. Когут П. І. Оптимізація в нелінійних еліптичних крайових задачах / П. І. Когут, О. П. Когут, О. А. Рядно. - Д. : ДДФА, 2010. 